\title{
ARTICLE
}

Clinical Study

\section{A prospective phase II study of pre-operative chemotherapy then short-course radiotherapy for high risk rectal cancer: COPERNICUS}

\author{
Simon Gollins ${ }^{1}$, Nicholas West ${ }^{2}$, David Sebag-Montefiore ${ }^{3}$, Shabbir Susnerwala ${ }^{4}$, Stephen Falk ${ }^{5}$, Nick Brown ${ }^{6}$, Mark Saunders ${ }^{7}$, \\ Philip Quirke ${ }^{2}$, Ruby Ray ${ }^{8}$, Philip Parsons ${ }^{9}$, Gareth Griffiths ${ }^{10}$, Tim Maughan ${ }^{11}$, Richard Adams ${ }^{8}$ and Chris Hurt (iD)
}

BACKGROUND: Neoadjuvant chemotherapy (NAC) allows earlier treatment of rectal cancer micro-metastases but is not standard of care. There are currently no biomarkers predicting long-term progression-free survival (PFS) benefit from NAC.

PATIENTS AND METHODS: In this single arm phase II trial, patients with non-metastatic magnetic resonance imaging (MRI)defined operable rectal adenocarcinoma at high risk of post-operative metastatic recurrence, received 8 weeks of oxaliplatin/ fluorouracil NAC then short-course preoperative radiotherapy (SCPRT) before immediate surgery. Sixteen weeks of post-operative adjuvant chemotherapy (AC) was planned. A pelvic MRI was performed at week 9 immediately post-NAC, before SCPRT. The primary end point was feasibility assessed by completion of protocol treatment up to and including surgery. Secondary endpoints included compliance, toxicity, downstaging efficacy, and PFS.

RESULTS: In total 60 patients were recruited May 2012-June 2014. In total 57 patients completed protocol treatment, meeting the primary endpoint. Compliance with NAC was much better than AC: Comparing NAC vs. AC, the median percentage dose intensity for fluoropyrimidine was $100 \%$ vs. $63 \%$ and for oxaliplatin $100 \%$ vs. $45 \%$. Treatment-related toxicity was acceptable with no treatment-related deaths. Post-NAC MRI showed 44 tumours (73\%) were T-downstaged and 22 (37\%) had excellent MRI tumour regression grade (mrTRG 1-2). Median follow-up was 27 months with 2-year PFS of $86.2 \%$ (10 events). On exploratory analysis, postNAC mrTRG predicted PFS with no event among those with excellent regression.

CONCLUSION: The regimen was well tolerated with effective downstaging and encouraging PFS. mrTRG response to NAC may be a new prognostic factor for long-term PFS, but needs validation in larger studies.

British Journal of Cancer (2018) 119:697-706; https://doi.org/10.1038/s41416-018-0209-4

\section{INTRODUCTION}

Approximately 15,000 patients are diagnosed with rectal cancer annually in the UK and surgery using total mesorectal excision (TME) is the standard of care. Using 'short-course' pre-operative radiotherapy (SCPRT) of 25 Gy over 5 working days, followed by TME within a week, reduces pelvic recurrence rates to approximately $5 \% .{ }^{1,2}$.

Local recurrence reduction has not impacted on distant metastatic relapse however, which is now the major cause of death. Histopathological features of resected specimens predict increased systemic recurrence risk including $>5 \mathrm{~mm}$ invasion of disease beyond the muscularis propria $(\geq \mathrm{T} 3 \mathrm{c})^{3,4}$ extra-mural venous invasion (EMVI) $)^{5}$ and lymph node involvement $(\mathrm{LN}+){ }^{6}$ With such features, distant metastatic relapse is $\sim 6$-fold greater than local recurrence. ${ }^{1,2,7}$ MRI scanning is the pre-treatment investigation which can most reliably identify such features. $^{8-10}$

Current UK practice, supported by NICE guidance ${ }^{11}$ is to complete local pelvic treatment with surgery \pm pre-operative radiotherapy, before considering systemic adjuvant chemotherapy (AC). However, a more recent meta-analysis of four trials incorporating preoperative radiotherapy suggested limited or no benefit for post-operative $A C$ in patients receiving pre-operative (chemo)radiotherapy. ${ }^{12}$ Using NAC as the first received treatment, allows earlier treatment of micrometastases and increased compliance. However, NAC has hitherto not been established as a standard of care in operable rectal cancer.

For tumours staged as operable, i.e., not threatening the surgical circumferential resection margin (CRM) on pre-treatment MRI, no downstaging is required prior to surgery and SCPRT

\footnotetext{
${ }^{1}$ North Wales Cancer Treatment Centre, Bodelwyddan, Denbighshire LL18 5UJ, UK; ${ }^{2}$ Pathology and Tumour Biology, Leeds Institute of Cancer and Pathology, University of Leeds, Leeds LS9 7TF, UK; ${ }^{3}$ Leeds Institute of Cancer and Pathology, University of Leeds and Leeds Cancer Centre, Leeds LS9 7TF, UK; ${ }^{4}$ Royal Preston Hospital, Fulwood, Preston PR2 9HT, UK; ${ }^{5}$ Bristol Haematology and Oncology Centre, University Hospitals Bristol NHS Foundation Trust, Bristol BS2 8ED, UK; ${ }^{6}$ Calderdale Royal Hospital, Salterhebble, Halifax HX3 OPW, UK; ${ }^{7}$ The Christie NHS Foundation Trust, Withington, Manchester M20 4BX, UK; ${ }^{8}$ Centre for Trials Research, Cardiff University, Room 409, Neuadd Meirionnydd, Heath Park, Cardiff CF14 4YS, UK; ${ }^{9}$ NCRI RTTQA, Velindre Cancer Centre, Velindre NHS Trust, Velindre Road, Cardiff CF14 2TL, UK; ${ }^{10}$ Southampton Clinical Trials Unit, Faculty of Medicine, Univeristy of Southampton, Tremona Road, Southampton SO16 6YD, UK and ${ }^{11}$ CRUK/MRC Oxford Institute for Radiation Oncology, University of Oxford, Old Road Campus Research Building, Roosevelt Drive, Oxford OX3 7DQ, UK

Correspondence: Simon Gollins (simon.gollins@wales.nhs.uk)
}

Received: 25 March 2018 Revised: 30 June 2018 Accepted: 9 July 2018 Published online: 17 August 2018 
followed by immediate surgery within a week, is a standard approach. ${ }^{1,2}$ This study (COPERNICUS: Chemotherapy then Radiation then Immediate Curative Surgery for operable rectal cancer) used 8 weeks of initial NAC with oxaliplatin and 5-Fluorouracil, then SCPRT prior to immediate surgery. It was a UK multicentre, open-label, single arm phase II trial in patients with MRI-staged operable rectal cancer at high risk of developing distant metastases post-operatively.

\section{METHODS}

Eligibility

Eligible patients were adults, ECOG Performance Status 0-1, with histopathologically confirmed rectal adenocarcinoma with the following features:

1. The inferior disease aspect was $\geq 4 \mathrm{~cm}$ from anal verge and the superior aspect was not more superior than the anterior S1/S2 interspace.

2. The mesorectal fascia was not threatened or involved, i.e., tumour was $>1 \mathrm{~mm}$ from mesorectal fascia on MRI.

3. The primary tumour was mrT3a-b (T3a: tumour invasion $\leq 1$ $\mathrm{mm}$ beyond muscularis propria; T3b: invasion $>1-5 \mathrm{~mm}$ ) in the presence of either EMVI or mesorectal lymph nodes(s)/ tumour deposit(s) of any size with irregular border or mixed signal intensity. Alternatively the primary tumour was $\mathrm{mrT3c}$ (invasion $>5-15 \mathrm{~mm}$ ) or T3d (invasion $>15 \mathrm{~mm}$ ) or T4 (invasion of visceral peritoneum for tumours with a component above peritoneal reflection was permitted although invasion of other organs was not), regardless of EMVI or nodal status. Low tumours did not involve levator ani or anal sphincters.

4. CT scan of chest and abdomen excluded metastatic disease.

Haematological, renal, and hepatic biochemical indices were satisfactory. All patients provided written informed consent to a medical doctor.

Treatment

NAC. Four, 14-day cycles of Oxaliplatin/5-Fluorouracil (OxMdG) were administered via a central venous catheter (day 1 oxaliplatin $85 \mathrm{mg} / \mathrm{m}^{2}$ plus levofolinic acid $175 \mathrm{mg}$ over $2 \mathrm{~h}$, then 5Fluorouracil $400 \mathrm{mg} / \mathrm{m}^{2}$ bolus, then 5-Fluorouracil $2400 \mathrm{mg} / \mathrm{m}^{2}$ continuous infusion over $46 \mathrm{~h}$ ).

Radiotherapy. Within 14 days following completion of the last 2weekly cycle of chemotherapy, i.e., within 15-28 days following the first day of the last cycle of chemotherapy, patients commenced treatment with SCPRT. Before commencing SCPRT, haematological and gastrointestinal toxicities should have resolved to $\mathrm{NCI} C T C A E \leq$ grade 1 .

COPERNICUS full Radiotherapy Guidelines are included in the Supplementary Material. Briefly, pelvic radiotherapy was planned using oral and intravenous contrast CT simulation with recommended $3 \mathrm{~mm}$ CT slices. $25 \mathrm{~Gy}$ in 5 daily fractions, prescribed according to recommendations of the International Commission on Radiation Units and Measurements (ICRU-50), was delivered over 5-7 days Monday to Friday as a 3D, conformally planned single-phase treatment, usually with four radiotherapy fields. Gross tumour volume (GTV) was defined using the diagnostic MRI scan and included all macroscopic tumour and any intervening normal rectal wall. Clinical target volume (CTV) was defined in two parts (CTVA and CTVB) and then combined to form the Final CTV (CTVF). CTVA consisted of GTV with a $1 \mathrm{~cm}$ margin grown in all directions. CTVB included the mesorectum, the presacral and internal iliac nodal structures. CTVB superior limit was the more superior of $2 \mathrm{~cm}$ above the most superior limit of GTV, or the S2/3 interspace. CTVB inferior limit was the more inferior of $2 \mathrm{~cm}$ inferior to the most inferior limit of GTV, or the superior limit of puborectalis. The final CTV (CTVF) was derived by combining CTVA and CTVB. The planning target volume (PTV) was derived by adding a $1 \mathrm{~cm}$ margin to CTVF in all directions. Verification with cone-beam CT or electronic portal imaging was used on the first 3 fractions of radiotherapy. Radiotherapy quality assurance was carried out via the UK National Cancer Research Institute Radiotherapy Trials Quality Assurance Group (NCRI RTTQA). Preaccrual quality assurance included completion of two benchmark cases: a delineation exercise and a planning exercise on a precontoured patient. These assessments were identical to those which had previously been defined for the ongoing UK phase III ARISTOTLE trial. Gaining previous approval for ARISTOTLE also conferred a centre with COPERNICUS approval. For on-trial, as a minimum, QA consisted of prospective individual case review (both contours and treatment plan) for the first patient from each radiotherapy delivery site. Radiotherapy Protocol compliance of the remaining patients was reviewed using a pre-designed Plan Assessment Form which captured clinically relevant dose-volume metrics.

Surgery. Surgery was recommended within 7 days of the last fraction of SCPRT although a gap of 14 days was acceptable.

AC. Between 6 and 8 weeks following the date of surgery, patients commenced eight, 14-day cycles of AC using OxMdG, although alternatively a combination of oxaliplatin and capecitabine could be used (a 14-day schedule of oxaliplatin $85 \mathrm{mg} / \mathrm{m}^{2} \mathrm{IV}$ day one followed by oral capecitabine at $1000 \mathrm{mg} / \mathrm{m}^{2}$ twice daily for 9 days).

Assessments

Toxicity was assessed as per US National Cancer Institute's Common Terminology Criteria for Adverse Events (CTCAE version 4.03) at the end of each treatment cycle and at the end of SCPRT. Capecitabine compliance was assessed by tablet count at each visit. An MRI was performed at 9 weeks (post NAC, pre-SCPRT) to assess downstaging and tumour regression grade (mrTRG) using a 5 -point scale. ${ }^{13}$ Resection specimens were evaluated by local histopathologists as per detailed trial-specific guidelines (see appendix $\mathrm{E}$ of the trial protocol, included as Supplementary Material). The 5-point Dworak system was used to assign a pathological tumour regression grade (pTRG), mirroring mrTRG. ${ }^{14}$ Sites sent formalin-fixed, paraffin-embedded blocks of pretreatment and surgical resection material to Leeds Institute of Cancer and Pathology, University of Leeds. All glass haematoxylin and eosin stained slides were also sent from the biopsy and resection specimens for scanning to create a permanent record (http://www.virtualpathology.leeds.ac.uk/clinical/colorectal/ copernicus) and facilitate central review of pTRG and tumour cell density (TCD) calculation. ${ }^{15}$

TNM Classification of Malignant Tumours 5th edition was used for histopathological staging. Histopathological substages T3a-d were defined similarly to those used for MRI. Pathological complete response (ypTOypN0) was confirmed as follows: Where tumour cells could not be found on the first assessment of up to five blocks of tumour, the whole area of the tumour/fibrotic scar was embedded and examined. If no tumour cells could be seen following assessment of these extra blocks, then three deeper levels were taken and examined from each tumour/fibrotic scar block. ypTOypNO was confirmed if no tumour cells were identified. There were no ypTON+cases.

$\mathrm{TCD}^{15}$ was measured by digitally scanning the glass haemotoxylin and eosin-stained slides at $\times 200$ magnification and annotating around the tumour. Approximately 300 random points within this area were then manually assessed to determine the underlying tissue components and calculate the TCD. For resected tumour TCD was expressed as either the 'whole tumour' TCD when the outlining included scar, or alternatively the area of the greatest 


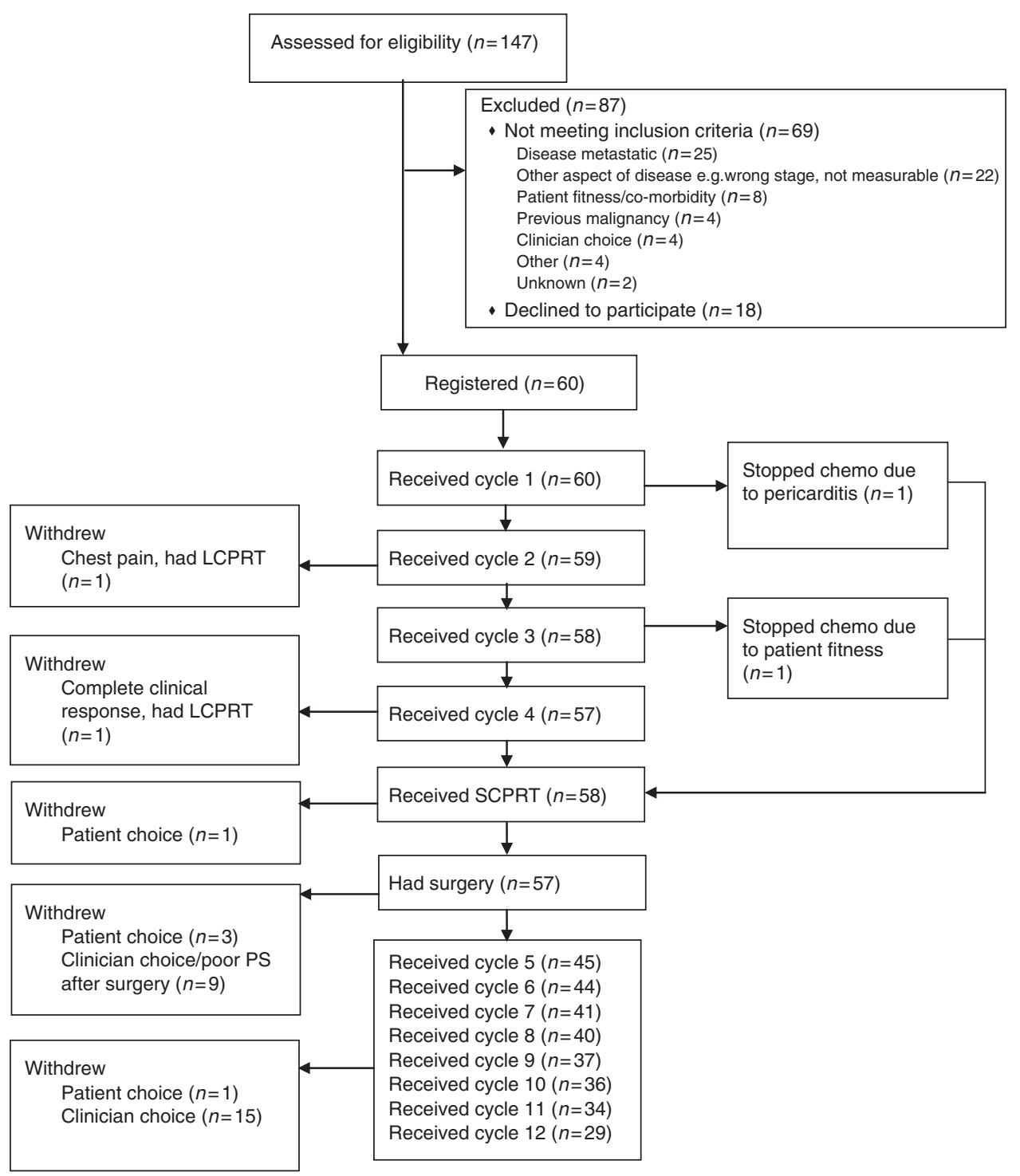

Fig. 1 Participant flow diagram

TCD when annotating a $3 \times 3 \mathrm{~mm}^{2}$ area in the region of apparent greatest residual tumour.

Post-operative morbidity was assessed at 30 days post-surgery. Patients were followed up to assess disease status at 6 months and 12 months following surgery, with a CT scan mandated at 12 months. Investigations and follow-up beyond 12 months were done as per institutional standard.

Statistics

This was a single arm phase II trial. The primary endpoint was completion of protocol treatment up to and including surgery defined as starting NAC, and completing SCPRT and surgery. If $>92 \%$ (as was found in $C R 07,{ }^{2}$ and not $<80 \%$, of patients completed surgery then it would warrant further investigation in the Phase III setting. Based on an $A^{\prime} H e r n$ design ${ }^{16}$ looking at proportion of patients completing surgery and setting $\mathrm{p} 0=0.80, \mathrm{p} 1=0.92,90 \%$ power, alpha $=0.1,57$ patients were required (with 50 patients completing surgery counting as success).

Secondary endpoints included compliance, toxicity, histopathological and radiological assessment of downstaging efficacy, and PFS. Exploratory analyses investigated the relationship between measures of downstaging efficacy and PFS.
Data were analysed according to a pre-specified analysis plan using the Stata SE 14 statistical package. All analyses were by intention to treat except toxicity analyses, which were conducted only in those patients who had some treatment during the related treatment phase, and the surgical complications analysis only in those who had surgery. Clopper-Pearson exact binomial method was used to calculate confidence intervals for the primary endpoint. We calculated \% of total dose (actual total dose divided by protocol total dose) and \% dose intensity (actual dose intensity [dose per unit time] divided by protocol dose intensity) for each protocol drug as measures of compliance. Baseline and presurgical weight was compared using a Wilcoxon signed rank test. Univariable ordinal regression was used to look for associations between categorical (number of baseline MRI risk factors (out of $\mathrm{T} \geq 3 \mathrm{c}$ or $\mathrm{N1}-2$ or $\mathrm{EMVI}+$ ), mrTRG, $\mathrm{pTRG}$,) and continuous variables (TCD). We calculated progression free survival (PFS) from date of registration to when a failure event (death, or confirmed evidence of distant metastases or loco-regional progression) occurred. Patients who were event-free were censored at the time they were last known to be event free. Follow-up time distribution was estimated using the reverse Kaplan-Meier method ${ }^{17}$ with patients censored at date of death or last trial assessment. We estimated event time distributions with the Kaplan-Meier method and 
looked for relationships between PFS and potential predictors using Cox regression in univariable models.

\section{RESULTS}

In total 60 patients were enroled from 14 UK centres between 25 May 2012 and 11 June 2014 (Fig. 1). At the time of analysis, all patients had completed at least 1 year of post-surgical follow-up or withdrawn from the study. Patient and tumour baseline characteristics are shown in Table 1. Pre-treatment MRI showed that $35(58 \%), 14(23 \%)$ and $11(18 \%)$ patients had 1,2 or 3 highrisk features, respectively.

\section{Toxicities}

Toxicities during neoadjuvant (end of cycle 1 to post SCPRT assessment) and adjuvant (end of cycle 5 to end of cycle 12) treatment periods are shown in Table 2 . The rate of any grade $3+$ toxicity was similar in each treatment period: neoadjuvant $24 / 60$ (40\%) and adjuvant 15/45 (33\%), with neutrophil count decrease being the most common toxicity in each case: 12/60 (20\%) and 5/ $45(11 \%)$, respectively. There were no deaths during treatment (including the post-surgical period). There was no evidence of a difference between baseline (median: $84.1 \mathrm{~kg}$, IQR: 78.1-92.1) and pre-surgical (median: $85.3 \mathrm{~kg}$, IQR: 76.1-92.7) weight $(z=0.796, p$ $=0.426, n=56$ ). Post-surgical complications within 30 days are shown in Supplementary Online Table 1 including three patients (5\%) who suffered an anastomotic dehiscence. Out of the 57 patients who had surgery, 49 (86\%) were discharged within 30 days, a median of 7 (IQR: 6-11) days after surgery.

\section{Compliance}

Compliance with NAC was much better than $A C$, as shown in Fig. 2. In total $45 / 60$ (75\%) of patients started AC. 22/60 (37\%) of patients switched from $5 \mathrm{FU}$ to capecitabine during $A C$. The median percentage total dose and dose intensity of 5FU/ capecitabine was 100 (IQR: 97-100) and 100 (IQR: 75-100) respectively during NAC and 80 (IQR: 5-88) and 63 (IQR: 5-81) respectively during $A C$. The median percentage total dose and dose intensity of oxaliplatin was 100 (IQR: 93-100) and 100 (IQR: 75-100) respectively during NAC and 58 (IQR: 0-98) and 45 (IQR: $0-77)$ respectively during $A C$.

Of the 58 patients who started SCPRT (see Fig. 1), the median time from the start of last cycle of NAC to the start of SCPRT was 24 days (IQR: 19-27, Range: 13-103), all patients received SCPRT at full protocol dose, and only 1 patient experienced a non-logistical delay during SCPRT (due to rheumatoid arthritis flare-up).

In total $57 / 60(95 \%, 90 \%$ Cls: $88-99)$ patients had surgery, the majority $(43,75 \%)$ had an anterior resection, $3(5 \%)$ a Hartmann's resection and 11 (19\%) an abdominoperineal excision. The median time from finishing pre-surgical treatment to having surgery was 7 days (IQR: $4-12$, Range: $1-34, n=57$ ). The median time from surgery to start of adjuvant chemotherapy was 63 days (IQR: 53-79, Range: 40-133, $n=45$ ). Three patients did not have surgery: one patient refused surgery because of anxiety with no recurrence at 18 months post-registration. Contrary to protocol, two patients received long-course chemoradiation rather than SCPRT. One of these later underwent a successful resection and in the other a 'watch and wait' approach was adopted with no recurrence at 17 months post-registration.

All 9 radiotherapy centres passed pre-accrual QA prior to entering patients into the trial. Of the 58 patients having SCPRT, the first patient's contours and plans from each radiotherapy centre underwent prospective review prior to radiotherapy starting. Only one centre was required to undergo repeat prospective review because they submitted their first patient's data for review after the patient had started their RT. One additional centre was requested to make some minor contouring amendments prior to RT-this was completed successfully and a
Table 1. Baseline characteristics and 9 week MRI results

\begin{tabular}{|c|c|c|}
\hline & $\begin{array}{l}\text { Baseline } \\
n(\%)^{\mathrm{a}}\end{array}$ & $\begin{array}{l}9 \text { weeks } \\
n(\%)^{\mathrm{a}}\end{array}$ \\
\hline \multicolumn{3}{|l|}{ Patients enroled } \\
\hline$N$ & 60 & \\
\hline \multicolumn{3}{|l|}{ Age (years) } \\
\hline Median (IQR, range, $n$ ) & $\begin{array}{l}63(56.5-70 \\
38-79,60)\end{array}$ & \\
\hline \multicolumn{3}{|l|}{ Gender } \\
\hline Male & $44(73.3)$ & \\
\hline Female & $16(26.7)$ & \\
\hline \multicolumn{3}{|l|}{ ECOG performance status } \\
\hline 0 & 55 (91.7) & \\
\hline 1 & $5(8.3)$ & \\
\hline \multicolumn{3}{|c|}{$\begin{array}{l}\text { Predominant differentiation } \\
\text { of primary tumour }\end{array}$} \\
\hline Well & $5(8.3)$ & \\
\hline Moderate & $49(81.7)$ & \\
\hline Poor & $2(3.3)$ & \\
\hline Unknown & $4(6.7)$ & \\
\hline \multicolumn{3}{|l|}{$\begin{array}{l}\text { Time from MRI scan to } \\
\text { registration (weeks) }\end{array}$} \\
\hline Median (IQR, range, $n$ ) & $\begin{array}{l}3.7(2.6-4.6 \\
0-5.9,60) \\
\text { before reg }\end{array}$ & $\begin{array}{l}9.4(8.7-10,7.4-12.4,58) \\
\text { after reg }\end{array}$ \\
\hline \multicolumn{3}{|l|}{$\begin{array}{l}\text { MRI-Craniocaudal length } \\
(\mathrm{mm})\end{array}$} \\
\hline Mean (SD, range, $n$ ) & $\begin{array}{l}49(11.9 \\
28-80,58)\end{array}$ & $34.3(13.5,7-70,52)$ \\
\hline \multicolumn{3}{|c|}{$\begin{array}{l}\text { MRI-Height from anal verge } \\
(\mathrm{mm})\end{array}$} \\
\hline Mean (SD, range, $n)$ & $\begin{array}{l}79(21.5 \\
40-140,58)\end{array}$ & $82.9(21.8,35-140,52)$ \\
\hline \multicolumn{3}{|l|}{ MRI T Stage } \\
\hline TO & & $4(7)$ \\
\hline $\mathrm{T} 1$ & & $1(2)$ \\
\hline $\mathrm{T} 2$ & $1(1.7)$ & $28(47)$ \\
\hline T3a & $17(28.3)$ & $6(10)$ \\
\hline $\mathrm{T} 3 \mathrm{~b}$ & $24(40)$ & $13(22)$ \\
\hline T3c & $14(23.3)$ & $4(7)$ \\
\hline T3d & $1(1.7)$ & $0(0)$ \\
\hline T4 & $3(5)$ & $0(0)$ \\
\hline Missing & $0(0)$ & $4(7)$ \\
\hline \multicolumn{3}{|l|}{ MRI N Stage } \\
\hline No & $7(11.7)$ & $38(63)$ \\
\hline N1 & $39(65)$ & $18(30)$ \\
\hline N2 & $14(23.3)$ & $2(3)$ \\
\hline Missing & $0(0)$ & $2(3)$ \\
\hline \multicolumn{3}{|l|}{ MRI M Stage } \\
\hline Mo & $60(100)$ & $58(97)$ \\
\hline Missing & $0(0)$ & $2(3)$ \\
\hline \multicolumn{3}{|l|}{ MRI-CRM involvement } \\
\hline Clear $(>1 \mathrm{~mm})$ & $59(98.3)$ & $57(95)$ \\
\hline Missing data & $1(1.7)$ & $2(3)$ \\
\hline \multicolumn{3}{|l|}{$\begin{array}{l}\text { MRI-Extramural venous } \\
\text { invasion }\end{array}$} \\
\hline Positive & 25 (41.7) & $9(15)$ \\
\hline
\end{tabular}






further patient review was not necessary. The remaining 48 plans were assessed using the Plan Assessment Form. All plans were considered acceptable.

Efficacy

Radiological downstaging. Table 1 shows the results from the post-NAC week $9 \mathrm{MRI}$ compared to baseline MRI. T Stage was downstaged in 44/60 (73\%), unchanged in 12/60 (20\%), and missing for $4 / 60(7 \%)$ of patients. N Stage was downstaged in 36/ $60(60 \%)$, unchanged in 22/60 (37\%), and missing for 2/60 (3\%) of patients. There was no recorded progression in either $\mathrm{T}$ or $\mathrm{N}$ stage during NAC. 22 tumours (37\%) showed excellent regression (mrTRG 1-2). EMVI reduced from 25/60 (42\%) pre-operatively to 9/ $60(15 \%)$ on the week 9 MRI.

Pathological downstaging. Surgical procedure information and pathology results are shown in Table 3. In total 7/57 (12\%) patients were found to have had a complete pathological response, confirmed by a central review that included a check of the number of deeper levels cut on the entirely embedded scars. Compared to baseline MRI, histopathological (ypT) T stage was downstaged in 42/57 (74\%), unchanged in 11/57 (19\%) and upstaged in 4/57 (7\%) of patients. Histopathological (ypN) N stage was downstaged in $37 / 57(65 \%)$, unchanged in $16 / 57(28 \%)$, and upstaged in $4 / 57(7 \%)$ of patients. The incidence of histopathological EMVI + in the resected specimen (11/57: 19\%) was similar

Table 2. CTCAE v4.02 grade $3+$ toxicity in patients during neoadjuvant (week 1 to post SCPRT assessment) and adjuvant (post cycle 5 to post cycle 12 assessment) treatment

\begin{tabular}{|c|c|c|c|}
\hline System organ class & Adverse event & Neoadjuvant $n(\%)^{\mathrm{a}}$ & Adjuvant $n(\%)^{\mathrm{b}}$ \\
\hline Any & Any & $24(40)$ & $15(33)$ \\
\hline Cardiac disorders & Pericarditis & $1(2)$ & $0(0)$ \\
\hline \multirow{4}{*}{ Gastrointestinal } & Diarrhoea & $1(2)$ & $3(7)$ \\
\hline & Dyspepsia & $1(2)$ & $0(0)$ \\
\hline & Rectal obstruction & $0(0)$ & $1(2)$ \\
\hline & Stomatitis & $2(3)$ & $0(0)$ \\
\hline \multirow[t]{2}{*}{ General disorders and administration site conditions } & Injection site reaction & $0(0)$ & $1(2)$ \\
\hline & Localised oedema & $1(2)$ & $0(0)$ \\
\hline Infections and infestations & Upper respiratory infection & $1(2)$ & $2(4)$ \\
\hline \multirow{2}{*}{ Investigations } & Weight loss & $0(0)$ & $1(2)$ \\
\hline & White blood cell decreased & $1(2)$ & $1(2)$ \\
\hline Metabolism and nutrition disorders & Hyperuricemia & $0(0)$ & $1(2)$ \\
\hline \multirow[t]{2}{*}{ Nervous system disorders } & Neuropathy & $1(2)$ & $0(0)$ \\
\hline & Syncope & $1(2)$ & $0(0)$ \\
\hline \multirow[t]{2}{*}{ Psychiatric disorders } & Agitation & $1(2)$ & $0(0)$ \\
\hline & Anxiety & $1(2)$ & $0(0)$ \\
\hline Respiratory, thoracic and mediastinal disorders & Cough & $1(2)$ & $0(0)$ \\
\hline \multirow[t]{2}{*}{ Skin and subcutaneous tissue disorders } & Palmar-plantar erythrodysesthesia syndrome & $0(0)$ & $1(2)$ \\
\hline & Rash & $1(2)$ & $0(0)$ \\
\hline Vascular disorders & Thromboembolic event & $2(3)$ & $0(0)$ \\
\hline
\end{tabular}





Fig. 2 Total dose and dose intensity curves for neoadjuvant and adjuvant chemotherapy

to that shown on the week 9 MRI (9/58: $16 \%$, Table 3).

Likewise waterfall plots of TCD showed a marked response (Supplementary Online Fig. 1). Median biopsy TCD was $37.2 \%$, reduced to $8.7 \%$ in resection whole $\mathrm{TCD}$.

Relationship between mrTRG and resection pathology. We found a significant association between mrTRG and PTRG $\left(x^{2}=26.5, p=\right.$ 0.048 ), resection greatest TCD (ordinal regression $z=-3.77, p<$ $0.001)$, and resection whole TCD $(z=-3.77, p<0.001)$ in the 54 patients with non-missing data.

Relationship between PTRG and TCD. Univariate ordinal regression showed no evidence of a relationship between PTRG and the number of baseline MRI risk factors $(z=-0.06, p=0.951)$ or biopsy $\operatorname{TCD}(z=0.29, p=0.774, n=57)$, but strong relationships between pTRG and resection greatest TCD $(z=-5.70, p<0.001, n=57)$, resection whole TCD $(z=-5.41, p<0.001, n=57)$, and resection whole TCD as a percentage of biopsy TCD $(z=-4.03, p<0.001$, $n=57)$.

PFS. The median follow-up for PFS was 27.3 months (IQR: 22.3-31.1). Of the 60 patients enroled in the trial, 10 had progression ( 8 distant ( 6 lung, 1 liver, 1 small bowel), and 2 local recurrences) at the time of analysis. The PFS rate at 2 years was 86.2\% (95\% Cls: 74.3-92.9). One patient died after 2.3 years.

Relationship between measures of downstaging and PFS. Strikingly, none of the 22 patients with an excellent response to NAC on the week 9 MRI (mrTRG 1-2) had a progression event. All 10 progression events occurred in the 34 patients with mrTRG 3-5 (Fig. 3a).

Univariate cox regression showed a weak association between pTRG (when split by complete/good vs moderate/minimal/none) and PFS (see Fig. $3 \mathrm{~b}$ ): $\mathrm{HR}=4.76$ (95\% Cls: $0.60-37.61, p=0.139$ ). When TCD was included as a continuous variable in a univariate cox regression, there was some evidence that resection greatest TCD was associated with worse PFS (HR 1.03, 95\% Cl: 1.00-1.07, $p$ $=0.066$ ) but evidence for association with other TCDs was weak: biopsy TCD HR 1.01 (95\% Cl: 0.96-1.06, $p=0.725)$, resection whole TCD HR 1.04 (95\% Cl: 0.99-1.10, $p=0.137$ ).

\section{DISCUSSION}

This study has demonstrated the safety and feasibility of introducing NAC prior to SCPRT and surgery. There was a strikingly better compliance to NAC versus $A C$, rates of Grade $3+$ toxicity were acceptable $(\leq 40 \%)$ and post-operative complications were similar to what might be expected with SCPRT alone followed by immediate surgery. ${ }^{2}$

In keeping with our findings a randomised phase II study (GCR3) of neoadjuvant versus post-operative adjuvant oxaliplatin and capecitabine in 108 patients treated with chemoradiation and surgery, demonstrated less toxicity $(p=0.0004)$ and better compliance $(p<0.0001)$ for NAC compared with AC. ${ }^{18}$

In attempting to successfully treat micro-metastases, which are the main cause of rectal cancer death, there is thus a strong rationale for assessing initial NAC in trials powered for 3-year PFS. A further potential benefit of NAC is earlier closure of temporary 
Table 3. Surgery and pathology

\begin{tabular}{|c|c|}
\hline & $n(\%)^{\mathrm{a}}$ \\
\hline \multicolumn{2}{|l|}{ Surgical procedure } \\
\hline Abdominoperineal excision & $11(19)$ \\
\hline Anterior resection & $42(74)$ \\
\hline Hartmann's & $3(5)$ \\
\hline $\begin{array}{l}\text { Anterior resection \& prophylatic TAH \& } \\
\text { BSO }\end{array}$ & $1(2)$ \\
\hline \multicolumn{2}{|l|}{$\begin{array}{l}\text { Does the patient have a defunctioning } \\
\text { stoma }\end{array}$} \\
\hline Yes & $43(75)$ \\
\hline No & $11(19)$ \\
\hline Missing & $3(5)$ \\
\hline \multicolumn{2}{|l|}{ If yes, what type? ${ }^{b}$} \\
\hline Ileostomy & $32(74)$ \\
\hline Colostomy & $10(23)$ \\
\hline Missing & $1(2)$ \\
\hline \multicolumn{2}{|l|}{ If yes, intention? ${ }^{b}$} \\
\hline Temporary & $29(67)$ \\
\hline Permanent & $14(33)$ \\
\hline \multicolumn{2}{|l|}{ Post-operative pathology } \\
\hline pTO & $7(12)$ \\
\hline $\mathrm{pT} 1$ & $3(5)$ \\
\hline $\mathrm{pT} 2$ & $19(33)$ \\
\hline pT3a & $9(16)$ \\
\hline pT3b & $10(18)$ \\
\hline pT3c & $8(14)$ \\
\hline pT4a & $1(2)$ \\
\hline pNO & $39(68)$ \\
\hline $\mathrm{pN} 1$ & $13(23)$ \\
\hline $\mathrm{pN} 2$ & $5(9)$ \\
\hline $\mathrm{RO}$ (>1 mm from margin) & $56(98)$ \\
\hline $\mathrm{R} 1$ ( $\leq 1 \mathrm{~mm}$ from margin) & $1(2)$ \\
\hline \multicolumn{2}{|l|}{ Number of lymph nodes examined } \\
\hline Median (IQR, range, $n$ ) & $19(14-25,1-48,57)$ \\
\hline \multicolumn{2}{|l|}{ Number of lymph nodes positive } \\
\hline 0 & $39(68)$ \\
\hline $1-2$ & $11(19)$ \\
\hline $3-5$ & $6(11)$ \\
\hline 13 & $1(2)$ \\
\hline \multicolumn{2}{|l|}{ Extramural venous invasion } \\
\hline Yes & $11(19)$ \\
\hline No & $46(81)$ \\
\hline \multicolumn{2}{|l|}{ Distance to CRM (mm) } \\
\hline Median (IQR, range, $n$ ) & $12(6-15.5,1-50,47)$ \\
\hline \multicolumn{2}{|l|}{ pTRG } \\
\hline No regression & $7(12)$ \\
\hline Minimal regression & $17(30)$ \\
\hline Moderate regression & $14(25)$ \\
\hline Good regression & $12(21)$ \\
\hline Complete regression & $7(12)$ \\
\hline \multicolumn{2}{|l|}{ Plane of resection of mesorectum } \\
\hline Muscularis propria & $2(4)$ \\
\hline Intramesorectal & $6(11)$ \\
\hline Mesorectal & $42(74)$ \\
\hline
\end{tabular}

\begin{tabular}{|c|c|}
\hline & $n(\%)^{a}$ \\
\hline Missing & $7(12)$ \\
\hline \multicolumn{2}{|c|}{ Plane of abdomnio-perineal excision ${ }^{c}$} \\
\hline Levator & $3(27)$ \\
\hline Sphincteric & $5(46)$ \\
\hline Intrasphincteric/perforated & 0 \\
\hline Missing & $3(27)$ \\
\hline \multicolumn{2}{|c|}{$\begin{array}{l}\text { Distance of tumour to distal surgical margin } \\
(\mathrm{mm})\end{array}$} \\
\hline Median (IQR, range, $n$ ) & $30(20-50,5-100,48)$ \\
\hline \multicolumn{2}{|l|}{ Involvement of distal margin? } \\
\hline No & $54(95)$ \\
\hline Missing & $3(5)$ \\
\hline \multicolumn{2}{|c|}{$\begin{array}{l}\text { Distance of tumour to proximal surgical } \\
\text { margin }(\mathrm{mm})\end{array}$} \\
\hline Median (IQR, range, $n$ ) & $\begin{array}{l}182.5(120-255,50-460, \\
46)\end{array}$ \\
\hline \multicolumn{2}{|c|}{ Involvement of proximal margin? } \\
\hline No & $53(93)$ \\
\hline Missing & $4(7)$ \\
\hline \multicolumn{2}{|c|}{ Is there any evidence of tumour perforation? } \\
\hline Yes & $2(4)$ \\
\hline No & $53(93)$ \\
\hline Unknown & $2(4)$ \\
\hline \multicolumn{2}{|c|}{ Peritoneal involvement? (ypT4b disease) } \\
\hline Yes & $1(2)$ \\
\hline No & $50(88)$ \\
\hline Missing & $6(11)$ \\
\hline \multicolumn{2}{|l|}{ Biopsy TCD } \\
\hline Median (IQR, range, $n$ ) & $\begin{array}{l}37.2(22.7-44.2,6.3-58.5 \text {, } \\
59)\end{array}$ \\
\hline \multicolumn{2}{|l|}{ Resection greatest TCD } \\
\hline Median (IQR, range, $n$ ) & $\begin{array}{l}21.4(2.7-39.1,0-59.9 \\
57)\end{array}$ \\
\hline \multicolumn{2}{|l|}{ Resection whole TCD } \\
\hline Median (IQR, range, $n$ ) & $8.7(1.3-16.1,0-38.2,57)$ \\
\hline \multicolumn{2}{|c|}{ Resection whole TCD as \% of biopsy TCD } \\
\hline Median (IQR, range, $n$ ) & $\begin{array}{l}19.4(3.2-53.8,0-468.9, \\
57)\end{array}$ \\
\hline $\begin{array}{l}\text { a unless otherwise indicated, } d \\
\text { those with defunctioning stor } \\
\text { abdominoperineal excision }\end{array}$ & $\begin{array}{l}{ }^{b} \text { denominator } N=43 \text {, i.e., } \\
N=11 \text {, i.e., those who had }\end{array}$ \\
\hline
\end{tabular}

stomas. The quality of surgery in the current study was good, with $74 \%$ of specimens resected in the mesorectal plane, suggesting that NAC then SCPRT is safe and not compromising surgical quality. The overall PFS rate at 2 years of $86.2 \%$ in this MRIselected high risk group of patients is encouraging.

Only one previous observational study examined NAC then SCPRT then surgery, recruiting 67 patients with CT3-4 tumours from 1997 to $2008 .^{19}$ Patients received eight weeks of NAC (5-FUbased for the first 28 patients then oxaliplatin-5FU for the subsequent 39 ), followed by SCPRT (20 Gy in five fractions) then surgery within the following week. Although histopathological downstaging was seen in $51 \%$ of patients, no pCR was reported.

In contrast the current regimen suggested greater anti-tumour activity, with superior T-downstaging and 7 tumours (12\%) showing a ypTOypN0 pCR. Reasons for this discrepancy may include the higher radiotherapy dose used in the current study (25 
Kaplan-Meier curve of PFS (months) by mrTRG

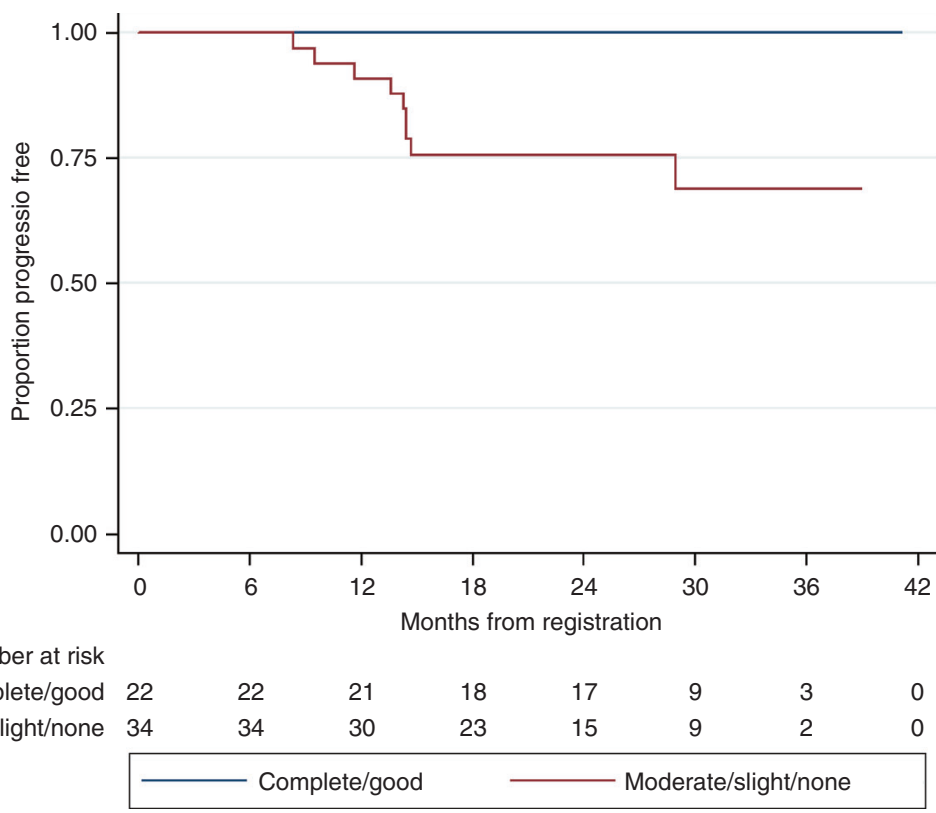

b

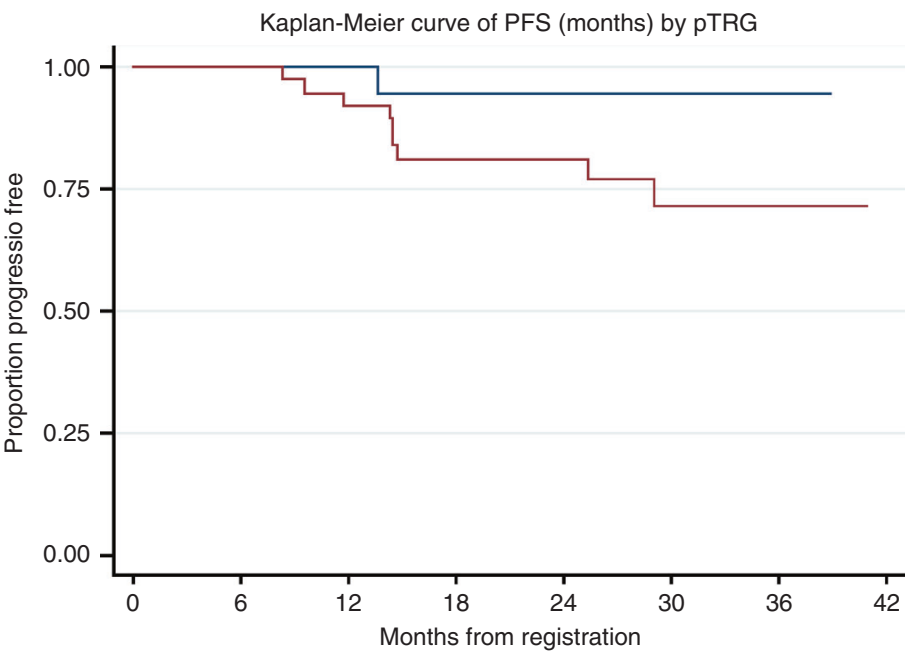

Number at risk

$\begin{array}{lllllllll}\text { Complete/good } & 19 & 19 & 19 & 16 & 15 & 5 & 1 & 0\end{array}$

Moderate/minimal/none

\begin{tabular}{llllllll|}
38 & 38 & 34 & 28 & 19 & 13 & 4 & 0 \\
\hline & Complete/good & & & Moderate/slight/none \\
\cline { 4 - 6 } & & & &
\end{tabular}

Fig. 3 a Kaplan-Meier curve of PFS (months) by mrTRG

vs. $20 \mathrm{~Gy}$ ), the use of both oxaliplatin and $5 \mathrm{FU}$ for all patients, and MRI selection (no MRI staging was used in Ciammella et al. ${ }^{19}$ ).

The Dutch TME trial failed to demonstrate any histopathological $T$ stage downstaging with SCPRT alone of $5 \times 5$ Gy followed by surgery within a week. ${ }^{20}$ However very significant downstaging was seen in the current study, so NAC appears of importance for the observed anti-tumour activity. The week $9 \mathrm{MRI}$ demonstrated conclusively that OxMdG NAC alone produced marked tumour downstaging.

The relative contributions of NAC and SCPRT to histopathological downstaging remain unclear and are potentially complex. Theoretical advantages to NAC before radiotherapy include improved tumour response through downstaging and increased oxygenation/radiosensitisation. Theoretical disadvantages include delayed radiotherapy and selection of radioresistant clones. ${ }^{21}$
There are currently no phase III studies examining NAC then SCPRT. The use of SCPRT for rectal cancer is decreasing in the UK. However, this trend could change dependent on the results of the phase III RAPIDO trial (NCT01558921), which completed accrual of 920 patients in June 2016 . This randomised patients with locally advanced rectal cancer to standard chemoradiation then surgery, versus an experimental arm of SCPRT followed by NAC then surgery.

A meta-analysis has highlighted limited efficacy of AC following preoperative radiotherapy. ${ }^{12}$ Several factors potentially reduce the effectiveness of $A C$ in rectal cancer. Morbidity from surgery and radiotherapy can delay initiation and reduces tolerance of $\mathrm{AC}$. In addition, the presence of post-operative stomas, expected in approximately $80 \%$ of patients, ${ }^{22}$ can impair or stop chemotherapy delivery altogether because of diarrhoea. ${ }^{23}$ The high response 
rate to initial NAC in the current study suggests that future comparison of pre- versus post-operative chemotherapy may be justified.

The UK has a high standard of routine pelvic MRI reporting following UK-wide initiatives such as the LOREC educational programme. ${ }^{24}$ MRI-defined TRG has been suggested to predict survival outcome post long-course chemoradiation ${ }^{13}$ and is increasingly reported in routine clinical practice following preoperative chemoradiation. Theoretically the baseline MRI scan could over-stage some patients in terms of their high-risk factors, leading to over-treatment. The loss of high-risk histopathological features in the eventually-resected specimen, due to the downstaging effect of neoadjuvant chemotherapy, makes if difficult to quantify this risk, although because of previously-reported strong correlation of MRI and histopathological high-risk features, we consider this risk low. ${ }^{8-10}$

Response to NAC assessed by MRI by RECIST criteria, was described in the EXPERT trials, which used 12 weeks oxaliplatin/ capecitabine before chemoradiotherapy and then surgery in 186 subjects. The radiological response rate was $63 \%$, and only 2 patients (1\%) progressed, ${ }^{25-27}$ echoing current findings. Pathological TRG following chemoradiation and surgery has recently been suggested to be both a prognostic factor and an individual patient level surrogate for disease-free survival within the randomised CAO/ARO/AIO-04 trial. $^{28}$

To our knowledge mrTRG has not been described in rectal cancer post-NAC alone, as in the current study. We found that mrTRG was well correlated with pTRG and resection TCD but better than them at predicting PFS, although a hazard ratio could not be calculated due to no events in the 22 excellent responders. The use of week 9 MRI post-NAC but before SCPRT most directly assessed the effect of NAC, without the addition of radiotherapy, which potentially affected subsequent pTRG and resection TCD. However, because of an overall limited number of events, it was not possible to perform multivariable regression to assess whether or not mrTRG remained a strong independent predictive marker for PFS, nor was it possible to assess whether or not it significantly outperforms other potential predictors such as pathological response.

Histopathological TCD provides a novel continuous measure of tumour regression, as opposed to the categorical pTRG measures, such as Dworak. Hitherto, TCD has not been reported in assessing rectal cancer response but here clearly demonstrated marked response, which correlated strongly with Dworak pTRG. Possibly relevant to future studies, the area of greatest residual TCD appeared to be more strongly associated with PFS than both whole tumour TCD (including scar) and pTRG. However, statistical power was limited due to the limited patient numbers and events.

In conclusion, at present there are no established biomarkers in patients receiving NAC that can predict those patients who may demonstrate a PFS benefit compared to those who will not. Our findings suggest that mrTRG following NAC may be a prognostic factor for PFS and are hypothesis-generating but need validation in future, larger studies. If confirmed, mrTRG has potential for use as a short-term surrogate in future studies of intensified NAC treatment strategies aimed at improving PFS in this high-risk group of patients.

\section{Availability of data and material}

The trial was sponsored by Cardiff University. Central trial coordination was by the Centre for Trials Research, Cardiff, including data collection and statistical analyses, where data supporting the results reported in the article can be found.

\section{ACKNOWLEDGEMENTS}

The Pathology and Tumour Biology laboratory at the University of Leeds is supported by grants from Yorkshire Cancer Research, the Pathological Society of Great Britain and Ireland, the Academy of Medical Sciences, The Medical Research Council and a National Institute of Health Research Senior Investigator Award. SG was a National Institute for Social Care and Health Research Academic Health Science Collaboration Clinical Research Fellow. We thank all the patients who participated in the trial and the research team from the participating centres. We thank members of the Independent Data Monitoring Committee and the Independent Trial Steering Committee for their oversight of the trial.

\section{AUTHOR CONTRIBUTIONS}

COPERNICUS was developed through and supported by the Anorectal Subgroup of the UK National Cancer Research Institute Colorectal Clinical Studies Group and was led by the trial management group composed of S.G., G.G., C.H., R.A., M.S., D.S.-M., P.Q., N.W., T.M. and R.R. C.H. was responsible for statistical analysis. P.P. and R.A. were responsible for pre-trial radiotherapy quality assurance. N.W. and P.Q. were responsible for tumour cell density assessment. S.S., S.F., and N.B. accrued significant numbers of patients and were involved in drafting and approving the final manuscript. C.H. and R.R. were responsible for the conduct of the trial, ensuring all required approvals were in place, and for collection and verification of the integrity of the data. Study results were interpreted by the trial management group; the trial management group also drafted the manuscript and collated the responses from all co-authors.

\section{ADDITIONAL INFORMATION}

Supplementary information is available for this paper at https://doi.org/10.1038/ s41416-018-0209-4.

Competing interests: Outside of the submitted work S.G. has received research funding from Roche and Pfizer. N.W. reports grants from Yorkshire Cancer Research, grants from Pathological Society of Great Britain and Ireland, during the conduct of the study; grants from Academy of Medical Sciences, grants from Cancer Research UK, outside the submitted work. N.B. reports speaking fees from Pfizer, and travel/ accommodation support from Roche. P.Q. reports personal fees and research funding from Amgen, personal fees and research funding from Roche, research funding from Ventana, during the conduct of the study; grants from Yorkshire Cancer Research programme grant, within and outside the submitted work and grants from Cancer Research UK, outside the submitted work. R.A. has received research funding from Cancer Research UK, personal fees and travel from Merck Serono, Amgen and Servier.

Ethics approval and consent to participate: The trial protocol, EudraCT No.: 2010 023083-40, was approved by the UK Medicines and Healthcare products Regulatory Agency and a UK Multi-Centre Research Ethics Committee (12/WA/0051), sponsored by Cardiff University and coordinated by the Centre for Trials Research at Cardiff University. All patients provided written informed consent before inclusion. The study was performed in accordance with the Declaration of Helsinki.

Consent for publication: All authors gave final approval of the version to be published.

Funding:: Funded by Cancer Research UK (ref. C23134/A11537).

\section{REFERENCES}

1. Peeters, K. C. et al. The TME trial after a median follow-up of 6 years: increased local control but no survival benefit in irradiated patients with resectable rectal carcinoma. Ann. Surg. 246, 693-701 (2007).

2. Sebag-Montefiore, D. et al. Preoperative radiotherapy versus selective postoperative chemoradiotherapy in patients with rectal cancer (MRC CR07 and NCICCTG C016): a multicentre, randomised trial. Lancet 373, 811-820 (2009).

3. Cawthorn, S. J. et al. Extent of mesorectal spread and involvement of lateral resection margin as prognostic factors after surgery for rectal cancer. Lancet $\mathbf{3 3 5}$, 1055-1059 (1990).

4. Willett, C. G., Badizadegan, K., Ancukiewicz, M. \& Shellito, P. C. Prognostic factors in stage T3N0 rectal cancer: do all patients require postoperative pelvic irradiation and chemotherapy? Dis. Colon Rectum 42, 167-173 (1999).

5. Talbot, I. C. et al. The clinical significance of invasion of veins by rectal cancer. $B r$. J. Surg. 67, 439-442 (1980).

6. Gunderson, L. L. et al. Impact of $\mathrm{T}$ and $\mathrm{N}$ stage and treatment on survival and relapse in adjuvant rectal cancer: a pooled analysis. J. Clin. Oncol. 22, 1785-1796 (2004). 
7. Rodel, C. et al. Oxaliplatin added to fluorouracil-based preoperative chemoradiotherapy and postoperative chemotherapy of locally advanced rectal cancer (the German CAO/ARO/AIO-04 study): final results of the multicentre, open-label, randomised, phase 3 trial. Lancet Oncol. 16, 979-989 (2015).

8. Beets-Tan, R. G. et al. Accuracy of magnetic resonance imaging in prediction of tumour-free resection margin in rectal cancer surgery. Lancet 357, 497-504 (2001).

9. Mercury Study Group. Diagnostic accuracy of preoperative magnetic resonance imaging in predicting curative resection of rectal cancer: prospective observational study. BMJ 333, 779 (2006).

10. Smith, N. \& Brown, G. Preoperative staging of rectal cancer. Acta Oncol. 47, 20-31 (2008).

11. National Institute for Health and Clinical Excellence. Colorectal Cancer: the Diagnosis and Management of Colorectal Cancer. http://guidance.nice.org.uk/ CG131 (2011).

12. Breugom, A. J. et al. Adjuvant chemotherapy after preoperative (chemo)radiotherapy and surgery for patients with rectal cancer: a systematic review and meta-analysis of individual patient data. Lancet Oncol. 16, 200-207 (2015).

13. Patel, U. B. et al. Magnetic resonance imaging-detected tumor response for locally advanced rectal cancer predicts survival outcomes: MERCURY experience. J. Clin. Oncol. 29, 3753-3760 (2011).

14. Dworak, O., Keilholz, L. \& Hoffmann, A. Pathological features of rectal cancer after preoperative radiochemotherapy. Int J. Colorectal Dis. 12, 19-23 (1997).

15. West, N. P. et al. The proportion of tumour cells is an independent predictor for survival in colorectal cancer patients. Br. J. Cancer 102, 1519-1523 (2010).

16. A'Hern, R. P. Sample size tables for exact single-stage phase II designs. Stat. Med. 20, 859-866 (2001).

17. Schemper, M. \& Smith, T. L. A note on quantifying follow-up in studies of failure time. Control Clin. Trials 17, 343-346 (1996).

18. Fernandez-Martos, C. et al. Phase II, randomized study of concomitant chemoradiotherapy followed by surgery and adjuvant capecitabine plus oxaliplatin (CAPOX) compared with induction CAPOX followed by concomitant chemoradiotherapy and surgery in magnetic resonance imaging-defined, locally advanced rectal cancer: Grupo cancer de recto 3 study. J. Clin. Oncol. 28, 859-865 (2010).

19. Ciammella, P. et al. Short-course preoperative radiotherapy combined with chemotherapy in resectable locally advanced rectal cancer: local control and quality of life. Radiol. Med. 118, 1397-1411 (2013).

20. Marijnen, C. A. et al. No downstaging after short-term preoperative radiotherapy in rectal cancer patients. J. Clin. Oncol. 19, 1976-1984 (2001).
21. Glynne-Jones, R., Anyamene, N., Moran, B. \& Harrison, M. Neoadjuvant chemotherapy in MRI-staged high-risk rectal cancer in addition to or as an alternative to preoperative chemoradiation? Ann. Oncol. 23, 2517-2526 (2012).

22. Healthcare Quality Improvement Partnership. National Bowel Cancer Audit Annual Report. https://www.hqip.org.uk/resources/national-bowel-cancer-auditannual-report-2016/ (2016).

23. Bosset, J. F. et al. Fluorouracil-based adjuvant chemotherapy after preoperative chemoradiotherapy in rectal cancer: long-term results of the EORTC 22921 randomised study. Lancet Oncol. 15, 184-190 (2014).

24. Dayal, S. \& Moran, B. LOREC: the English Low Rectal Cancer National Development Programme. Br. J. Hosp. Med. 74, 377-380 (2013).

25. Chau, I. et al. Neoadjuvant capecitabine and oxaliplatin followed by synchronous chemoradiation and total mesorectal excision in magnetic resonance imagingdefined poor-risk rectal cancer. J. Clin. Oncol. 24, 668-674 (2006).

26. Chua, Y. J. et al. Neoadjuvant capecitabine and oxaliplatin before chemoradiotherapy and total mesorectal excision in MRI-defined poor-risk rectal cancer: a phase 2 trial. Lancet Oncol. 11, 241-248 (2010).

27. Dewdney, A. et al. Multicenter randomized phase II clinical trial comparing neoadjuvant oxaliplatin, capecitabine, and preoperative radiotherapy with or without cetuximab followed by total mesorectal excision in patients with highrisk rectal cancer (EXPERT-C). J. Clin. Oncol. 30, 1620-1627 (2012).

28. Fokas E., et al. Tumor regression grading after preoperative chemoradiotherapy as a prognostic factor and individual-level surrogate for disease-free survival in rectal cancer. J. Natl Cancer Inst. 109 https://doi.org/10.1093/jnci/djx095 (2017).

Open Access This article is licensed under a Creative Commons Attribution 4.0 International License, which permits use, sharing, adaptation, distribution and reproduction in any medium or format, as long as you give appropriate credit to the original author(s) and the source, provide a link to the Creative Commons license, and indicate if changes were made. The images or other third party material in this article are included in the article's Creative Commons license, unless indicated otherwise in a credit line to the material. If material is not included in the article's Creative Commons license and your intended use is not permitted by statutory regulation or exceeds the permitted use, you will need to obtain permission directly from the copyright holder. To view a copy of this license, visit http://creativecommons. org/licenses/by/4.0/.

(c) The Author(s) 2018 